\title{
Recent Advances in Anticancer Drugs Development: G-Quadruplex as New Drug Target
}

\section{Prashansa Agrawal ${ }^{*}$}

Department of Chemistry, Case Western Reserve University, Cleveland, Ohio, 44106, USA

Nucleic Acids are large biomolecules and indispensable for life. They include deoxyribonucleic acid (DNA) and ribonucleic acid (RNA). Nucleic acids can adopt distinct non-canonical, highly compact secondary structure, G-quadruplex, in the dynamic region of chromosomal DNA and RNA transcripts, predominantly in telomeric sequences, and in promoter region of numerous genes including oncogenes such as Bcl-2 [1,2], VEGF [3,4], c-myc [5]. There are 376,000 putative quadruplex sequences (PQS) in the human genome that have been identified through genome-wide surveys based on a quadruplex folding rule [6], however, all of them may not exist in vivo. Recently, the existence of DNA G-quadruplex has been visualized on chromosomes in human cells [7]. These G-quadruplexes are an active target of drug discovery. The DNA G-quadruplexes formed in the promoter region of oncogene have been shown to be potential targets for anticancer drugs $[8,9]$ and proteins. The formation of these quadruplexes in telomeres has been shown to regulate the activity of the enzyme telomerase, which maintains the length of telomeres and is involved in $\sim 85 \%$ of all cancers. For example, Telomestatin [10,11], S2T1-6OTD (telomestatin synthetic Derivative) [12], SYUIQ-5 [13] interact with G-quadruplex formed in telomere and myc sequences and show their inhibitory activity in cancer cell growth. This has become a progressively large field of research.

The G-quadruplexes are very condensed structures and formed by the ganosine (G)-rich DNA and RNA sequences and consists of several stacked G-tetrads. Each G-tetrad has four guanine arranged in a square planar arrangement and held together by hoogsteen hydrogen bonding. Besides this, each G-quadruplex structure is further stabilized by the presence of a monovalent cation, mainly potassium, which is localized in the center between each pair of tetrads. The G-quadruplexes could affect gene activity either by upregulation or down regulation, which can be achieved by inducing or stabilizing G-quadruplex formation through a G-quadruplex interacting molecule (small molecule drugs or protein) that can stabilize the G-quadruplex structure and thus perform their desired function [8]. The validation of drug-targeted G-quadruplex DNA and the modulation of cancer genes' expression has been intensely increased in the recent past, thus opening new avenue for cancer research.

Though a lot of research has been focused on DNA G-quadruplexes, there has lately been a rapid advancement in the area of RNA G-quadruplexes, chiefly in the $5^{\prime}$-UTRs (untranslated regions) of mRNAs. RNA G-quadruplexes in the 5'-UTRs of mRNAs impact posttranscriptional regulation of gene expression, which affects disruption of normal cell behavior in human diseases, particularly cancers. The recent in-vitro study on small molecule G-quadruplex binding compound that can selectively target RNA G-quadruplexes exposed a new and striking opportunity for RNA-directed drug design [14]. However, there is still a major challenge to understand the systematic effects and selectivity in in-vivo environment. It is distinct that the RNA G-quadruplex motif embodies a structurally attractive scaffold for small molecule targeting and therefore provides a productive area for future research [15].

In addition to DNA and RNA, G-quadruplexes can be formed from a modified RNA nucleotide called locked nucleic acid (LNA). This is also stated as inaccessible RNA. Using LNA based oligonucleotides for therapeutic purpose is an evolving field in biopharmaceutical industry. The Danish pharmaceutical company Santaris Pharma owns the solitary rights to therapeutic uses of LNA technology [16]. They are now developing a new LNA based, hepatitis $\mathrm{C}$ drug called miravirsen, targeting miR-122, which is in Phase II clinical testing [17]. There is another artificially synthesized polymer similar to DNA or RNA, called Peptide nucleic acid (PNA) which also can form G-quarduplex. They do not occur naturally but PNA oligomers have been used in recent years in molecular biology procedures, diagnostic assays and antisense therapies.

First-in-class G-quadruplex-interacting compound in vivo that has reached Phase II clinical trials for the treatment of neuroendocrine/ carcinoid tumors is Quarfloxin (also known as CX-3543 or itarnafloxin), developed by Cylene Pharmaceuticals [18]. It is a fluoroquinolonebased antitumor agent derived from norfloxin via A-62176 and QQ58. Quarfloxin interrupts the interaction between the nucleolin protein and the G-quadruplex DNA structure in the ribosomal DNA, which results in the inhibition of ribosome synthesis and tumor cell apoptosis.

DNA G-quadruplexes, besides being a potential drug target, have also been potential cancer therapeutics. AS1411 (Antisoma, London, UK) is a guanosine-rich 26-base G-quadruplex-forming oligonucleotide aptamer that can induce apoptosis and thus inhibit the growth of malignant cells, is presently under Phase II clinical trials for the cure of renal cancer and acute myeloid leukemia. This aptamer has been shown to have cancer-selective antiproliferative activity against a variety of malignant tumors [19]. Another G-quadruplexbearing oligonucleotides aptamer is Anti-thrombin aptamer, TBA (also known as G15D, HTQ, HD1 or ARC183) is a guanosine-rich 15 -mer single-stranded DNA. It has been used in the coronary artery bypass graft surgery as a short-term anti-coagulant. Its optimized form (NU172) is now under phase II clinical trial by ARCA Biopharma (NCT00808964) [20]. In addition, antibodies are also used for targeting the G-quadruplex. A high-affinity single-chain antibody has been developed which is extremely specific for antiparallel telomeric repeats from Stylonychia lemnae macronuclei and binds to the telomeric repeats in vivo [21].

The design and synthesis of new high-affinity G-quadruplex interacting drugs will provide a new molecular probe for cancer

${ }^{*}$ Corresponding author: Prashansa Agrawal, Department of Chemistry, Case Western Reserve University, Cleveland, Ohio, 44106, USA, Tel: +1 216-368-2404; E-mail: prashansa.agrawal@case.edu

Received March 23, 2015; Accepted March 23, 2015; Published March 27, 2015

Citation: Agrawal P (2015) Recent Advances in Anticancer Drugs Development: G-Quadruplex as New Drug Target. J Pharmacovigilance 3: e134. doi: 10.4172/2329$6887.1000 \mathrm{e} 134$

Copyright: (c) 2015 Agrawal P. This is an open-access article distributed under the terms of the Creative Commons Attribution License, which permits unrestricted use, distribution, and reproduction in any medium, provided the original author and source are credited. 
Citation: Agrawal P (2015) Recent Advances in Anticancer Drugs Development: G-Quadruplex as New Drug Target. J Pharmacovigilance 3: e134. doi: 10.4172/2329$6887.1000 \mathrm{e} 134$

therapy. Drugs which bind to and stabilize G-quadruplexes can be used to suppress the elongation of telomeres and the gene transcription and translation of oncogenes, which will result in senescence and apoptosis of cancer cells. Besides naturally occurring nucleic acids G-quadruplexes, guanine-rich oligonucleotide aptamers are also able to adopt different kinds of stable G-quadruplex conformation and show a highly specific binding affinity to their target molecules, analogous to antibodies. They have shown to induce cell death in cancer cells and significant anticancer activity in preclinical and clinical studies. Thus, the G-quadruplex can be target as well as tool in anticancer drug development and therefore can be a very novel object for cancer therapy.

\section{References}

1. Dai J, Chen D, Jones RA, Hurley LH, Yang D (2006) NMR solution structure of the major G-quadruplex structure formed in the human BCL2 promoter region. Nucleic Acids Res 34: 5133-5144.

2. Agrawal P, Lin C, Mathad RI, Carver M, Yang D (2014) The Major G-Quadruplex Formed in the Human BCL-2 Proximal Promoter Adopts a Parallel Structure with a 13-nt Loop in K (+) Solution. J Am Chem Soc 136: 1750-1753.

3. Sun D, Guo K, Rusche JJ, Hurley LH (2005) Facilitation of a structural transition in the polypurine/polypyrimidine tract within the proximal promoter region of the human VEGF gene by the presence of potassium and G-quadruplex-interactive agents. Nucleic Acids Res 33: 6070-6080.

4. Agrawal P, Hatzakis E, Guo K, Carver M, Yang D (2013) Solution structure of the major G-quadruplex formed in the human VEGF promoter in $\mathrm{K}^{+}$: Insights into loop interactions of the parallel G-quadruplexes. Nucleic Acids Res 41 : 10584-10592.

5. Yang D, Hurley LH (2006) Structure of the biologically relevant G-quadruplex in the C-MYC promoter. Nucleosides Nucleotides Nucleic Acids Res 25: 951-968.

6. Huppert JL, Balasubramanian S (2005) Prevalence of quadruplexes in the human genome. Nucleic Acids Res 33: 2908-2916.

7. Biffi G, Tannahill D, McCafferty J, Balasubramanian S (2013) Quantitative visualization of DNA G-quadruplex structures in human cells. Nature Chem 5:182-186
8. Balasubramanian S, Hurley LH, Neidle S (2011) Targeting G-quadruplexes in gene promoters: a novel anticancer strategy? Nat Rev Drug Discov 10: 261275

9. Brooks TA, Hurley LH (2010) Targeting MYC expression through G-quadruplexes. Genes Cancer 1: 641-649.

10. Shammas MA, Shmookler Reis RJ, Li C, Koley H, Hurley LH, et al. (2004) Telomerase inhibition and cell growth arrest after telomestatin treatment in multiple myeloma. Clin Cancer Res 10: 770-776.

11. Miyazaki T, Pan Y, Joshi K, Purohit D, Hu B, et al. (2012) Telomestatin impairs glioma stem cell survival and growth through the disruption of telomeric G-quadruplex and inhibition of the proto-oncogene, c-Myb. Clin. Cancer Res 18: $1268-1280$.

12. Shalaby $T$, von Bueren $A O$, Hurlimann $M L$, Fiaschetti $G$, Castelletti $D$, et al. (2010) Disabling c-Myc in childhood medulloblastoma and atypical teratoid/ rhabdoid tumor cells by the potent G-quadruplex interactive agent S2T16OTD. Mol Cancer Ther 9: 167-179.

13. Zhou WJ, Deng R, Zhang XY, Feng GK, Gu LQ, et al. (2009) G-quadruplex ligand SYUIQ-5 induces autophagy by telomere damage and TRF2 delocalization in cancer cells. Mol Cancer Ther 8: 3203-3213.

14. Patel DJ, Phan AT, Kuryavyi V (2007) Human telomere, oncogenic promoter and 5'-UTR G-quadruplexes: Diverse higher order DNA and RNA targets for cancer therapeutics. Nucleic Acids Res 35: 7429-7455.

15. Burge S, Parkinson GN, Hazel P, Todd AK, Neidle S (2006) Quadruplex DNA: Sequence, topology and structure. Nucleic Acids Res 34: 5402-5415.

16. Developing LNA technology for new-generation cancer drugs. SP2 Magazine. March 2006.

17. Franciscus A (2010) Hepatitis C Treatments in Current Development. HCV Advocate.

18. Duan W, Rangan A, Vankayalapati H, Kim MY, Zeng Q, et al. (2001) Design and synthesis of fluoroquinophenoxazines that interact with human telomeric G-quadruplexes and their biological effects. Mol Cancer Ther 1: 103-120.

19. Bates PJ, Laber DA, Miller DM, Thomas SD, Trent JO (2009) Discovery and development of the G-rich oligonucleotide AS1411 as a novel treatment for cancer. Exp Mol Pathol 86: 151-164.

20. Study of NU172 as Anticoagulation in Patients Undergoing Off-pump CABG Surgery (SNAP-CABG-OFF). Clinical Trials gov.

21. Fernando $H$, Rodriguez $R$, Balasubramanian $S$ (2008) Selective recognition of a DNA G-quadruplex by an engineered antibody. Biochemistry 47: 9365-9371. 